\title{
The Inclusion of decentralized and self-organized system in the process of construction of design thinking
}

SIGRADI2018 TECHNOPOLITICAS

xxii congresso da sociedade iberoamericana de gráfica digital 22th conference of the iberoamerican society of digital graphics 07|08|09|novembro|2018 iau usp | são carlos | sp br

\author{
Adriana Edith Granero \\ UBA-FADU/UB-FAU | Argentina | adriana.granero@gmail.com
}

\begin{abstract}
This work exposes the possible composition of a system composed of "crowd-working" of static, inert, flexible architecture elements, similar or identical entities, the "tesserae" and the integration with the link generated with Artificial Intelligence artifacts, a complex adaptive system, as a first experimental step to developments of Nanomaterials and systems that respond to the construction of the projective thought of the architectural envelope. The research responds to a general strategy of theoretical revision, with inductive and mixed methods. The exploration work examines the relative space within the idea of reason and the social function of architecture.
\end{abstract}

Keywords: Self-organized; Decentralized; Nanorrobotic; Parametrism; Architectural Envelope

\section{INTRODUCCIÓN}

Existe una estrecha relación entre la forma de la arquitectura y la sustentabilidad desde el punto de vista de la eficiencia y optimización. Pero también existe una estrecha relación entre la arquitectura, el hombre y las actividades humanas. La necesidad de alojamiento y cobijo, ponen en evidencia el vínculo, el clivaje con la supervivencia de ser humano, su relación con sus necesidades emocionales y prácticas. La importancia de la arquitectura es la de proteger la vida del hombre y proteger el planeta. Podemos inferir que la envolvente arquitectónica es la tercera piel que envuelve al hombre y de esta manera, podemos entender el aspecto de la envolvente arquitectónica como una prolongación de esta, con su composición y su funcionamiento. Dichas características y propiedades nos inducen a enmarcar esta investigación dentro de la biomimética. Intrínsecamente en este marco, la arquitectura y la forma de la envolvente tienen un papel que es esencialmente simbólico.

Desde hace unos años hemos visto la aplicación de la biomimética y la robótica en las superficies de las construcciones arquitectónicas transformando a la arquitectura de estática en dinámica, está última combina a la arquitectura y a la ciencia de la computación en un crossing over del que participa el diseño y la tecnología. Existen construcciones tipo Transformers, son las que se basan en movimiento dinámico de sus partes, accionadas automáticamente y con movimiento programado que no depende de la fuerza de la gravedad. Coexisten otras cuyos movimientos dinámicos dependen de las fuerzas de la naturaleza y no mecánicas como Kinetics Art en este caso Kinetics Architecture. Las construcciones que utilizan mecatrónica tienen en su mayoría programas escritos que establecen qué tipo de actividad realizarán de acuerdo registro obtenido por sensores o bien sus movimientos son supeditados al resto del proyecto, son como un velo o una piel superpuesta y aparentemente no integrada al pensamiento proyectual.

Mientras que esta investigación propone una arquitectura cuya forma se adapte a situaciones cambiantes en su entorno y para ello se propone la integración de un sistema de inteligencia de enjambre que se basa en el movimiento dinámico con más grado de libertad que los producidos hasta la actualidad, el crossing over y la coordinación del sistema en agentes (robots) sensores/actuadores que produzcan movimientos ondulantes similares a los peristálticos por analogía para la producción de la homotermia como parte de la evolución de la superficie que encierran los espacios arquitectónicos vivibles.

Este concepto genera una forzada modificación en la escala y la constitución de los elementos arquitectura que conforman la superficie exterior. Pero además, se modifica el concepto de espacio arquitectónico estático, deja de ser un espacio vivencial con límites exactos.

\section{ANTECEDENTES}

Haciendo un poco de historia y en lo que se refiere a la biomimética, podemos nombrar la obra de varios arquitectos entre ellos de Antonio Gaudí, denominada como arquitectura biomórfica (Estévez, 2015) y por aproximación a la aplicación de la analogía de la naturaleza al habitat de la huanidad. Podemos decir que Antonio Gaudí investigó y trabajó en el primer nivel de la biomimética (Benyus, 2009) reproduciendo las formas 
naturales. Más recientemente henos encontrado el trabajo de equipos multidisciplinarios que investigaron y trabajaron en el segundo nivel de la biomimética como en Eastgate Center, Kinetics Architecture, Kinetics Facade, Climate adaptive Skins y Built Dynamic Facade, construcciones en las que según algunos de los autores, se trabajó imitando los procesos naturales. En la mayoría de los casos los fundamentos los encontramos en el reino vegetal y animal especialmente con la biomimética de procesos que se refiere especialmente al comportamiento especial de algunas plantas vasculares dentro del reino vegetal y que corresponde al movimiento de nastia y especialmente la foto nastia y la termo nastia. Algunos de estos ejemplos poseen un control electrónico de motores, otros placas de aluminio, en otros casos tienen un sistema externo de sobreado PTFE, también encontramos ejemplos con la utilización de láminas de fibra de carbono, o aquellos con mecanismos y sensores que funcionan como una pupila o un obturador y otros accionan con el viento de manera natural.

\section{ANÁLISIS DE ANTECEDENTES EN ARQUITECTURA}

En la primera parte de la investigación se han buscado ejemplos construídos de arquitectura biomimética estática y artículada, natural y mecánica. Se han seleccionado algunos de los ejemplos encontrados que son una muestra representativa del estado del arte en la actualidad. A continuación se expresa una breve reseña con características relevantes y al final (Tabla 1) una tabla comparativa:

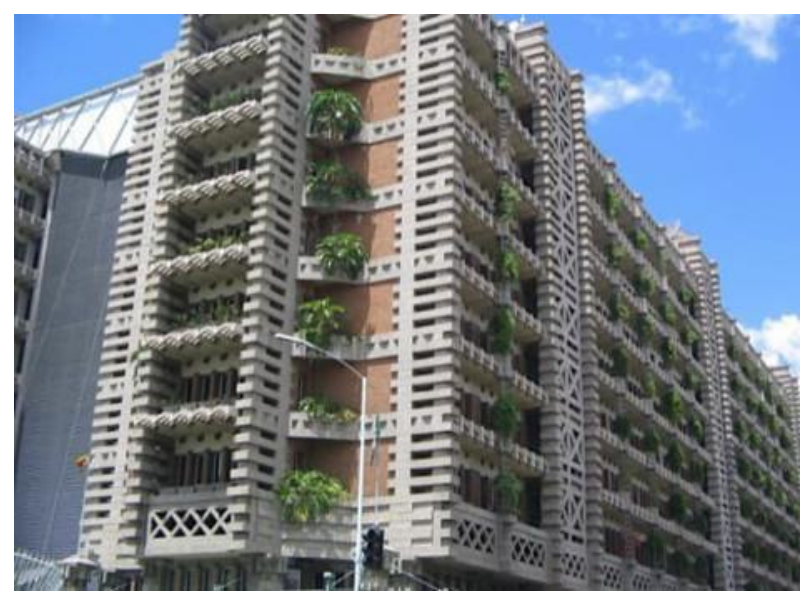

Figura 1: Eastgate Centre - Michael Pearce

Fuente: http://www.lboro.ac.uk/service/publicity/newsreleases/2004/04_100-termites.html

Eastgate Center (Figura 1) es un edificio que alberga un centro comercial y oficinas, es un ejemplo de biomimética con la técnica utilizada por las termitas (artrópodo) en la realización de sus nido, es un edificio ventilado y climatizado por medios naturales. Partió del proyecto TERMES de la Universidad de Loughborough en 2004, Leicestershire, Reino Unido. Trabajo de investigación de biólogos e ingnieros civiles o en construcción.

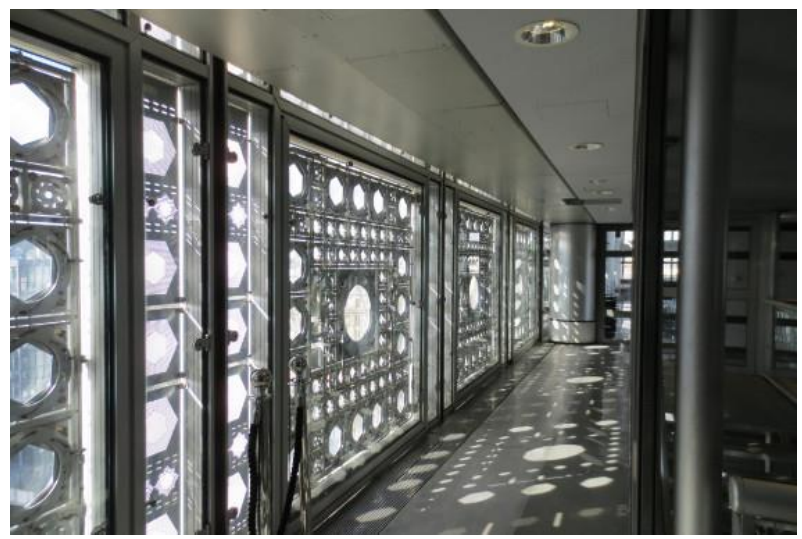

Figura 2: Instituto del Mundo Arabe - Jean Nouvel, Architecture Studio, Gilbert Lezenes, Pierre Soria. Fuente:

https://es. wikiarquitectura.com/edificio/instituto-del-mundoarabe/\#lg=1\&slide $=1$

El Instituto del Mundo Árabe (Figura 2): edificio y fachada que responden a la cultura árabe y magnifica los elementos del arquetipo de esa cultura, el tratamiento de la luz con bastidores y filtros, con una serie tramas con figuras geométricas superpuestas. En la fachada meridional estás tramas con figuras geométricas se funden en diafragmas "Moucharabiehs" que permiten el manejo de la luz, las transparencias, los reflejos, las refracciones y los efectos a contraluz. Las ventanas del IMA poseen celulas fotoeléctricas de diferente tamaño para su mecanización generando de esta manera el control de la luminosidad de manera automática y generando un juego de luz y sombra en su interior vinculado a la cultura árabe. Imitan en Fototropismo (negativo).

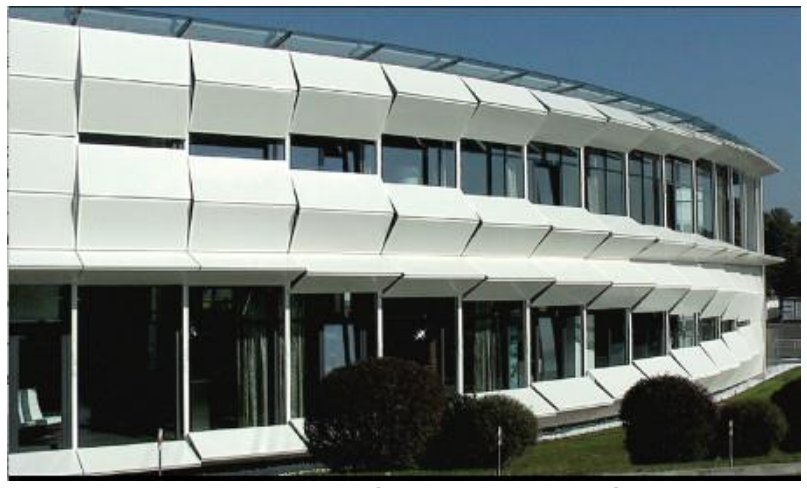

Figura 3: Kiefer Technic Showroom - Ernst Giselbrecht Fuente: https://www.archdaily.com/89270/kiefer-technicshowroom-ernst-giselbrecht-partner

Kiefer Technic Showroom (Figura 3), es un edificio de oficinas y espacio expositivo con una fachada dinámica mecánica que cambia las condiciones exteriores, optimiza el clima interno y permite a los usuarios personalizar sus propios espacios con controles de usuario. La fachada está compuesta por una estructura de aluminio con espacio para realizar tareas de mantenimiento a los motores y partes móviles que la componen que funcionan como pantalla solar y está formada por paneles realizados en aluminio. 


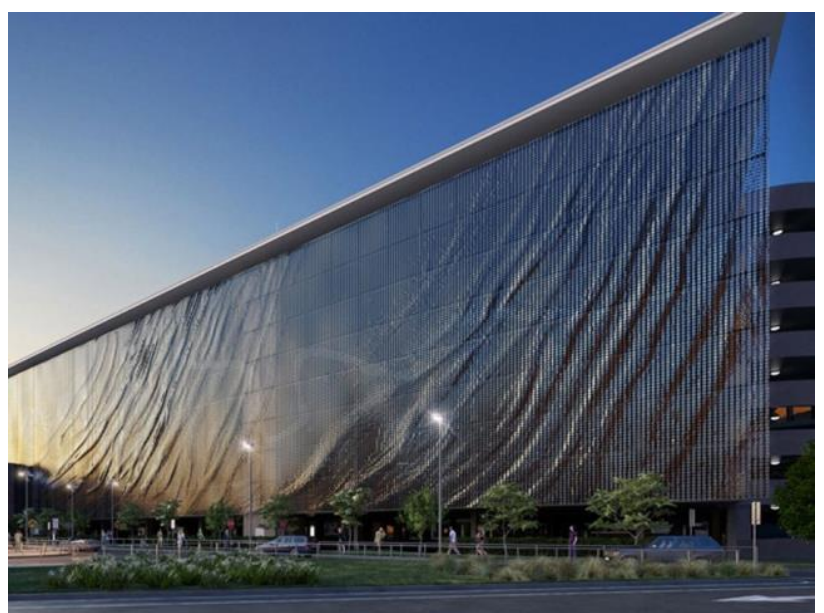

Figura 4: Brishbane Airport - UAP + Ned Kahn - Fuente: https://www.frameweb.com/news/brisbane-airport-kinetic-parking garage-fa

El Brishbane Airport (Figura 4) es un estacionamiento con fachada dinámica eólica y por gravedad, compuesta por aproximadamente 250.000 paneles de aluminio suspendidos (117.000 en su página), generando un diálogo directo entre lo construído y lo natural. El proyecto crea también beneficios ambientales como la sombre y la ventilación natural. Además de varios beneficios que se relacionan con el mejoramiento estético, el juego de luz y sombra sobre paredes y piso dentro del estacionamiento como el efecto de contemplación desde el exterior.

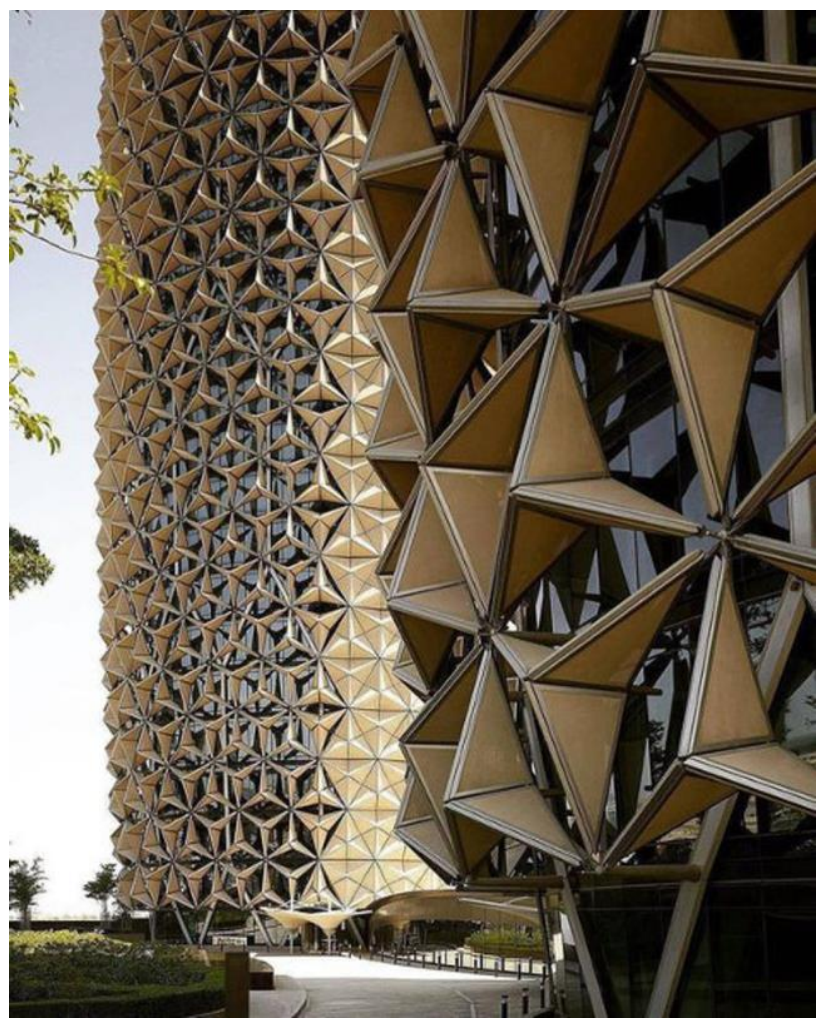

Figura 5: Al Bahar Towers Facade - AEDAS - Fuente: https://es. wikiarquitectura.com/edificio/torres-albahar/\#lg=1\&slide=14

El edificio Al Bahar Towers Facade (Figura 5) fue resultado del concepto de diseño adecuado cultural y ambientalmente y cumple con las aspiraciones del Plan de Desarrollo del 2030 para Abu Dhabi, las Torres Al Bahar cuentan con dos sistema de detección avanzado que fue diseñado para integrar el edificio con su contexto cultural y responder directamente a las necesidades climáticas de la región: la forma de "mashrabiya" de las pantallas, anclada en la tradición arquitectónica islámica de Oriente Medio y el movimiento dinámico de cada una de las unidades individuales que recuerda la respuesta de las plantas nativas. Imitación del comportamiento de las plantas: Fotonastia.

Gallery Center City - UNStudio (Figura 6) es un edificio con fachada dinámica de doble capa que hace uso de la ilusión óptica y el camuflaje (el camaleón) para esconder la escala y generar la sensación de imágenes dobles.

One Ocean Kinectic Facade (Figura 7), fue el proyecto ganador de la competencia internacional abierta en 2009, pone de manifiesto la biología cambiante de la arquitectura, la relación natural y biomimética con el entorno no fue buscada visualmente sino que se incorporó por los materiales, polímero reforzado con fibras de vidrio. Imitan branquias.

Sede Q1 (Figura 8), este edificio fue creado con el concepto de reducir la radiación solar directa y el deslumbramiento, mejoramiento del rendimiento y del confort, en respuesta se diseñó una fachada de parasoles o sombrillas con 400.000 láminas de acero inoxidable en forma de rejilla trapezoidal o triangular unidas a una estructura vertical con controles automáticos y sensores fotosensibles que trasmiten las señales de manera independiente a cada motor y generan el movimiento de cada parasol o sombrilla de manera individual. Nuevamente se imita la propiedad fototrópica negativa de algunas especies vegetales.

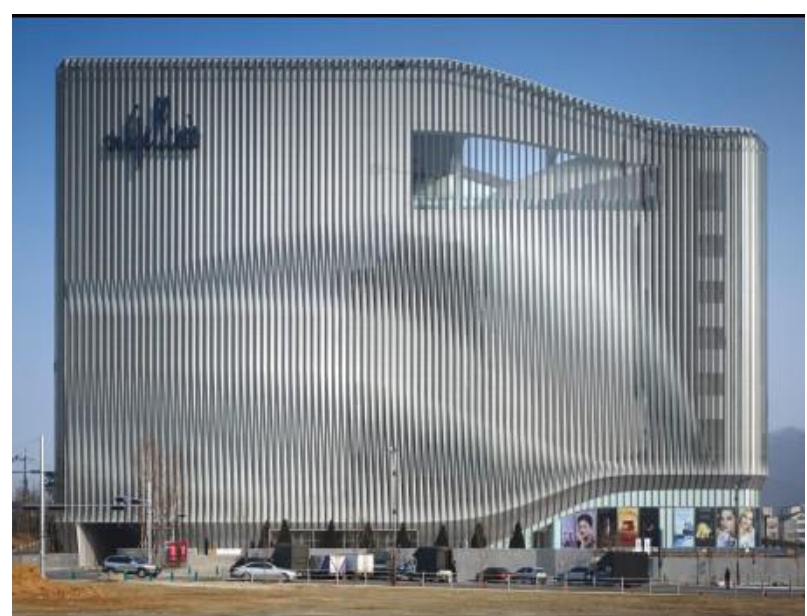

Figura 6: Galería Center City - UNStudio - Fuente: https://www.unstudio.com/en/page/388/galleria-centercity

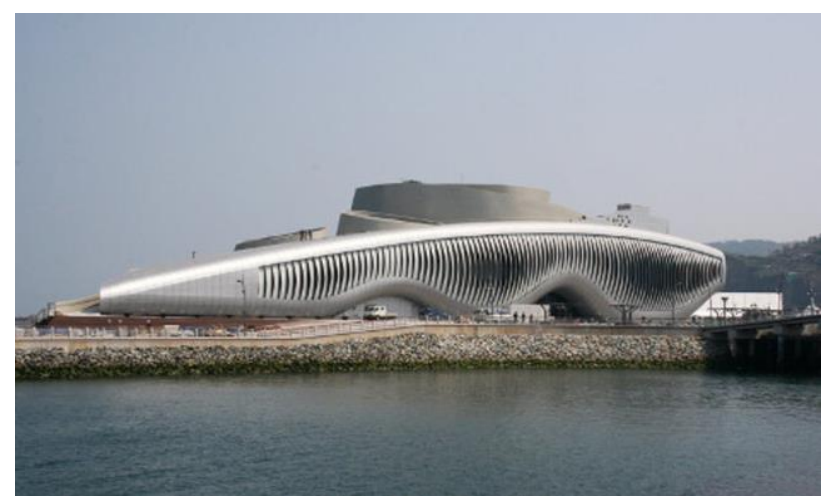

Figura 7: One Oceans Kinetic Facade - SOMA - Fuente: http://www.soma-

architecture.com/index.php?page=theme_pavilion\&parent=2 


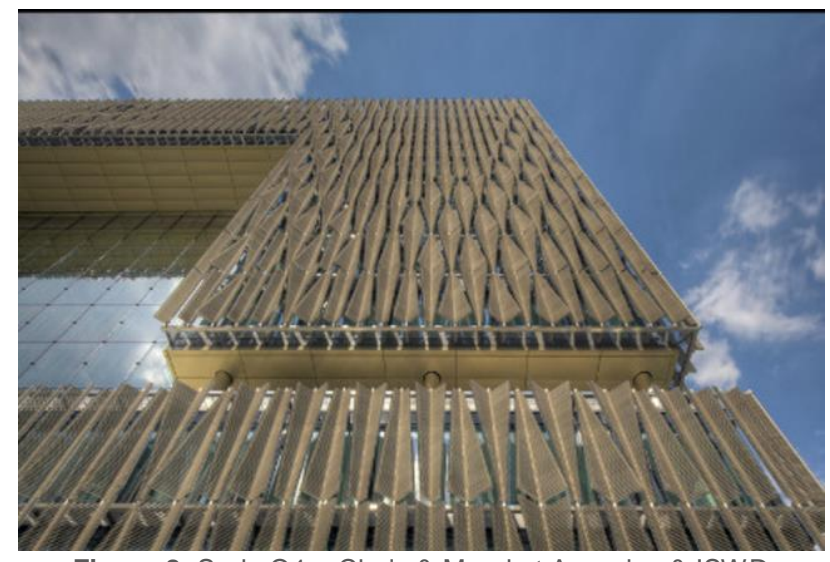

Figura 8: Sede Q1 - Chaix \& Morel et Associes \& ISWD Architekten - Fuente: https://www. plataformaarquitectura.cl/cl/02 235832/q1-thyssenkrupp-quarter-essen-jswd-architekten-chaixmorel-et-associes
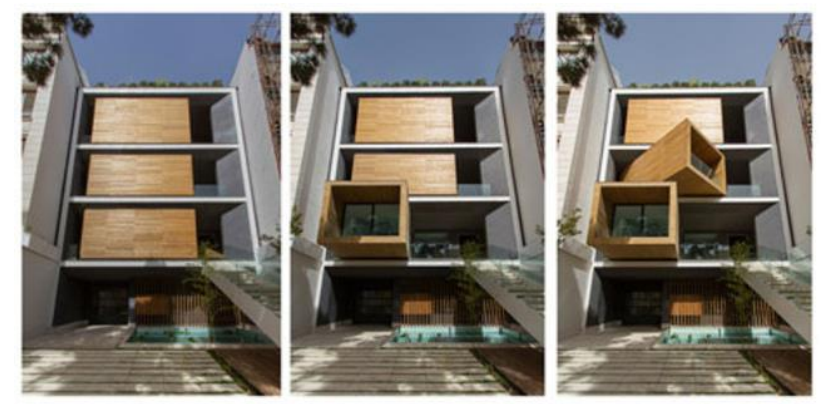

Figura 9: Sharifi-Ha House - dRMM Fuente: http://nextoffice.ir/\#!/project/sharifi-ha-house/

Sharif-Ha House (Figura 9 y 10), este proyecto ha dado un paso más en el desarrollo del mejoramiento en función a la eficiencia energética y confort. Ha buscado a través de una forma tridimensional y flexible dar respuesta a estas premisas, para ello se han diseñado una serie de volúmenes móviles, otros fijos, una estructura y un vacío. Imita el comportamiento de Heliotropismo que encontramos en especial en Helianthos Annuus.
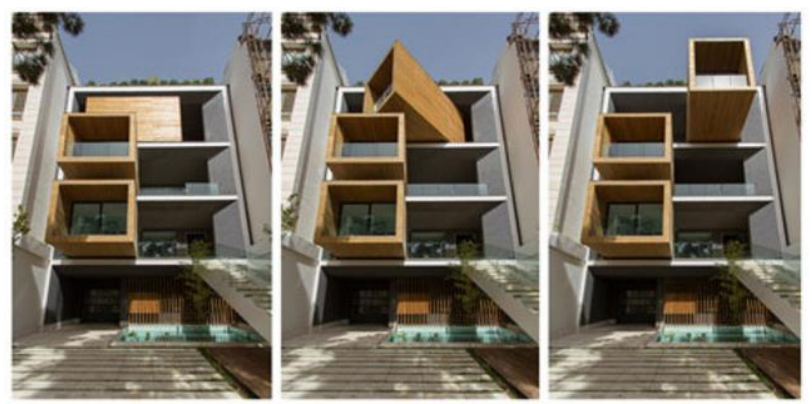

Figura 10: Sharifi-Ha House - dRMM - Fuente:

http://nextoffice.ir/\#!/project/sharifi-ha-house/

SDU Campus Kolding (Figura 11), al igual que varios de los nombrados anteriormente, imita el movimiento de Fototropismo - (negativo) para dar respuesta a condiciones climáticas variables, posee un sistema de sombreado en la fachada con sensores para controlar los niveles de calor e iluminación y evitar así el rendimiento deficiente por sobrecalentamiento o la ausencia del confort visual por el deslumbramiento. En este caso el diseño consta de 1.600 aletas triangulares de acero perforado, que al igual que otros ejemplos se controlan mecánicamente con motores. Se ha buscado en este caso un efecto secundario mediante la utilización del color, imitando el comportamiento de las aves al desplegar y mostrar su plumaje en el momento del apareamiento, creando de esta menera un foco de atención y una toma de decisión.

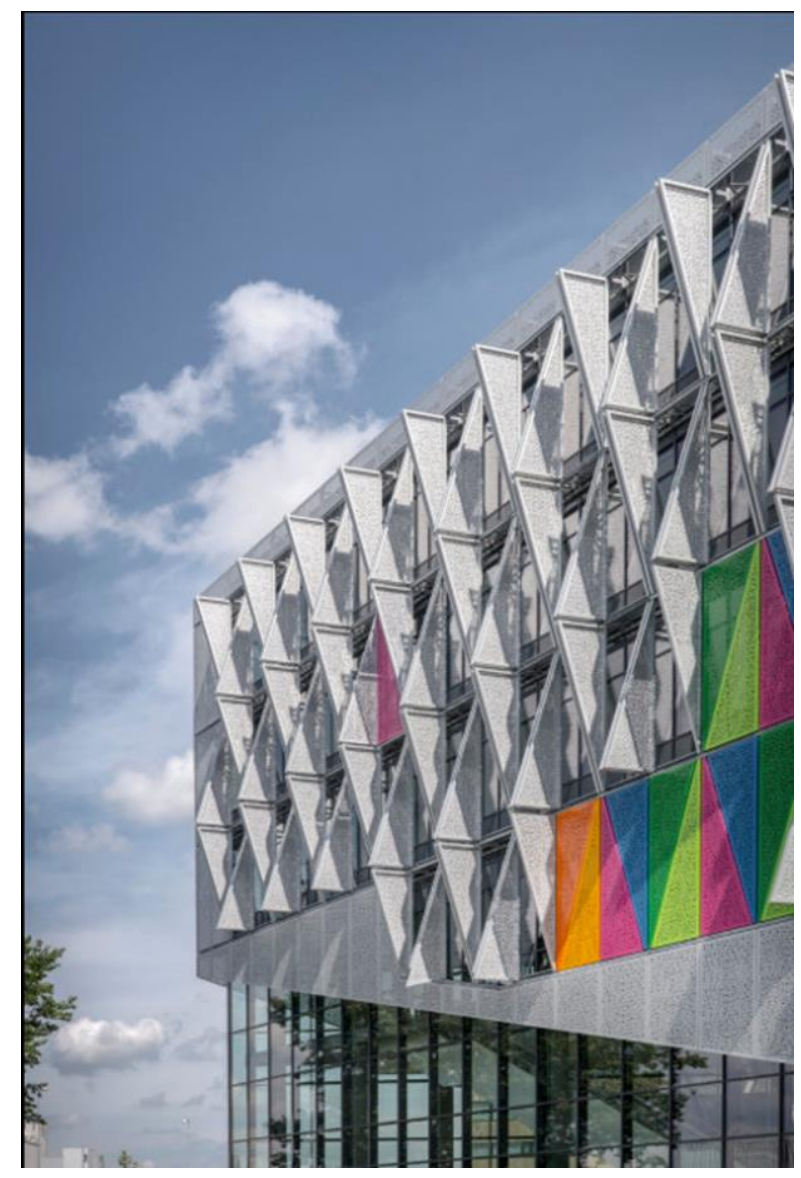

Figura 11: SDU Campus Kolding - Henning Larsen Architects Fuente: https://henninglarsen.com/en/projects/featured/0942-sducampus-kolding

Tabla 1: Síntesis del análisis de los antecedentes arquitectónicos encontrados en la siguiente tabla:

\begin{tabular}{|c|c|c|c|c|}
\hline Obra & Autor/es & Año & Sistema & Reproduce \\
\hline $\begin{array}{l}\text { Eastgate } \\
\text { Center }\end{array}$ & $\begin{array}{l}\text { Michael } \\
\text { Pearce }\end{array}$ & 2004 & Natural & $\begin{array}{l}\text { Técnica de } \\
\text { Artrópodo }\end{array}$ \\
\hline IMA & $\begin{array}{c}\text { Jean } \\
\text { Nouvel, el } \\
\text { al }\end{array}$ & 2007 & Mecánico & $\begin{array}{c}\text { Fototropismo } \\
(-)\end{array}$ \\
\hline $\begin{array}{l}\text { Kiefer } \\
\text { Technic } \\
\text { Showroom }\end{array}$ & $\begin{array}{c}\text { Ernest } \\
\text { Giselbretch }\end{array}$ & & Mecánico & $\begin{array}{c}\text { Fototropismo } \\
(-)\end{array}$ \\
\hline $\begin{array}{l}\text { Brishbane } \\
\text { Airport }\end{array}$ & $\begin{array}{l}\text { UAP+Ned } \\
\text { Kahn }\end{array}$ & & Natural & $\begin{array}{c}\text { Dinámica } \\
\text { Eólica }\end{array}$ \\
\hline $\begin{array}{l}\text { Al Bahar } \\
\text { Tower } \\
\text { Facade }\end{array}$ & AEDAS & & Mecánica & Foto nastia \\
\hline $\begin{array}{l}\text { Galería } \\
\text { Center } \\
\text { City }\end{array}$ & UNStudio & & Mecánica & Camuflaje \\
\hline $\begin{array}{l}\text { One } \\
\text { Ocean } \\
\text { Facade }\end{array}$ & SOMA & 2009 & Mecánica & $\begin{array}{l}\text { Órgano } \\
\text { respiratorio de } \\
\text { animales } \\
\text { acuáticos }\end{array}$ \\
\hline Sede Q1 & $\begin{array}{c}\text { Chaix \& } \\
\text { Morel, et al }\end{array}$ & & Mecánica & $\begin{array}{c}\text { Fototropismo } \\
(-)\end{array}$ \\
\hline $\begin{array}{l}\text { Sherif-Ha } \\
\text { House }\end{array}$ & dRMM & & Mecánica & Heliotropismo \\
\hline $\begin{array}{l}\text { SDU } \\
\text { Campus } \\
\text { Holding }\end{array}$ & $\begin{array}{c}\text { Hennig } \\
\text { Larsen } \\
\text { Architects }\end{array}$ & & Mecánica & $\begin{array}{c}\text { Fototropismo } \\
(-)\end{array}$ \\
\hline
\end{tabular}




\section{ANTECEDENTES EN ROBOTICA}

Desde otro área del conocimiento existen investigaciones que se conectan con esta investigación, tal es el caso de CWRU Biorobots Lab. En 2011 (University, 2011) presentó un desarrollo que se basó en el movimiento de las lombrices, filo anélida y perteneciente al reino animal que viajan contrayendo sus segmentos corporales secuencialmente. Este método de locomoción es particularmente efectivo en espacios restringidos. Los robots de gusano blando pueden eventualmente tener aplicación en la inspección de tuberías, excavación o exploración. Comprender la física y el control de este movimiento es un problema que abordaron con modelos mecánicos, matemáticos y de simulación por computadora.

Han desarrollado varios diseños para un robot que usa la peristalsis, el mismo método usado por las lombrices de tierra y otras especies del reino animal. Este prototipo utiliza una malla exterior trenzada para producir ondas de movimiento a lo largo del cuerpo del robot.

La Malla Modular Compatible con la Lombríz (CMMWorm, Compatible Modular Mesh Worm) (Figura 12 y 13), utiliza una malla obediente accionada en segmentos modulares para crear formas de onda a lo largo de su cuerpo. Estas formas de onda pueden generar un movimiento peristáltico del similar al del cuerpo de una lombriz. La malla modular está construida con piezas impresas en 3D printer disponibles comercialmente, lo que permite probar la variedad de que los componentes se pueden intercambiar fácilmente. Además de tener segmentos controlados independientemente y propiedades de malla intercambiables, CMMWorm también tiene un alto rango de contracción (52\% del diámetro máximo). El robot de seis segmentos puede atravesar superficies planas y tuberías.

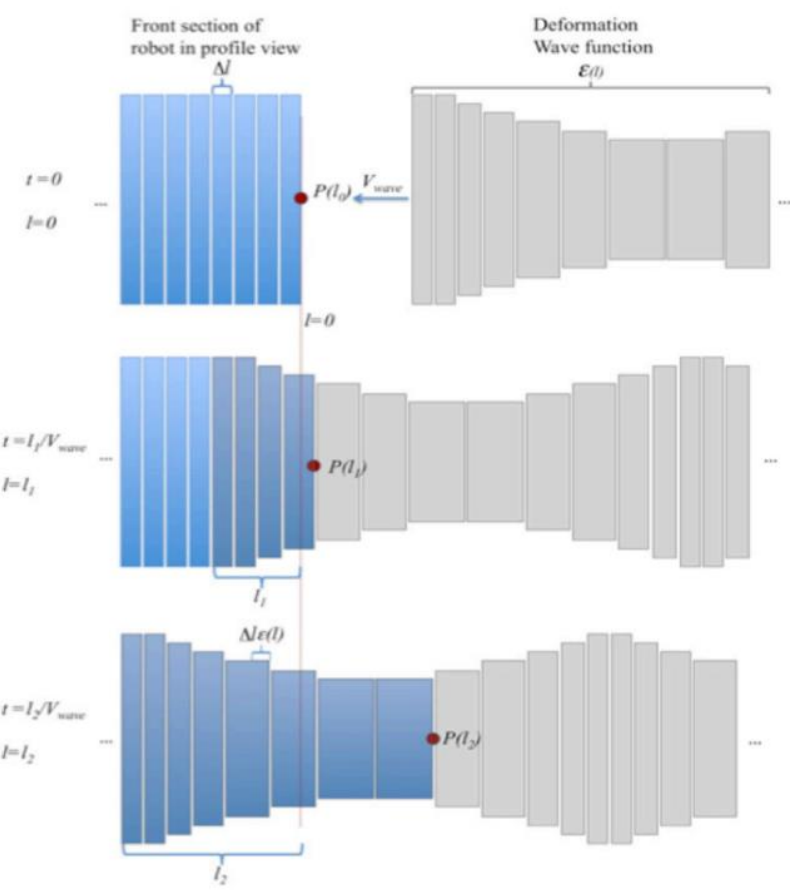

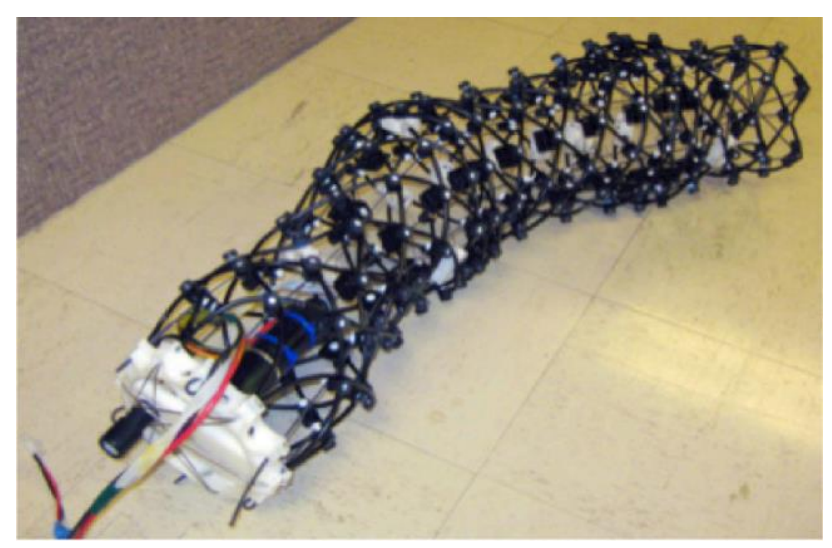

Figura 12 y 13: CMMWorm - Fuente:

http://biorobots.case.edu/projects/softworm/

Una de las carácterísticas a utilizar y que ha desarrollado para el robot que usa la peristalsis, se fundamenta en el modelo de Wison-Cowan (Neurons, 1972 ) que pertenece a la neurociencia computacional y que describe las interacciones entre una población de neuronas simples exitadoras e inhibidoras y la respuesta a los estímulos.

\section{PROPUESTA METODOLÓGICA}

En trabajos de investigación realizados previamente se ha puesto de manifiesto que una envolvente biomimética puede ser interpretada: como la envolvente arquitectónica que utiliza el estudio del funcionamiento de los sistemas y conductas de la forma natural y lo reproduce. En dicha oportunidad se expresa que todos los seres vivos de la naturaleza han tenido que adaptarse con continuos cambios en un proceso evolutivo para no desaparecer y se debatió sobre la necesidad de búsqueda de un elemento de arquitectura que pertenezca a la tetradimensión (Granero, El espacio arquitectónico topológico digital, 2016), que facilitara la adecuación absoluta de la envolvente del espacio arquitectónico en tiempo real. Como continuación de esas dos investigaciones, el presente trabajo expone un análisis de antecedentes con revisión teórica en otras áreas del conocimiento y la exploración con análisis descriptivos. Haciendo una analogía con especies de la naturaleza y su hábitat, estudiando la evolución de las mismas; de su estudio hemos encontrado que, en los Artrópodos, el filo Moluscos, los Anélidos, los Reptiles y el Hombre, las especies más numerosas y diversas del reino animal, poseen características que en su estudio posiblemente permitirán elaborar respuestas de confort y de rendimiento.

En los Artrópodos (Hickman, 2006), encontramos que su exterior consta de un exoesqueleto que está formado por su sistema tegumentario, una cutícula compuesta por quitina y que además está combinado por segmentos y apéndices articulados. Los segmentos se fusionan en grupos según sus funciones y se denominan tagma.

El concepto de exoesqueleto ya ha sido estudiado y analizado desde tiempos remotos, desde los egipcios, pasando por la edad media se lo utilizó en forma de armadura para eventos bélicos y con la función defensiva, por supervivencia como un elemento superpuesto, como un elemento que imitaba la protección para perpetuar la especie. 
Hay antecedentes de exoesqueletos artificiales mecánicos que poseen el funcionamiento con sensores biométricos y actúan en fracción de segundos, es el caso de HAL-3 (2000) y HAL-5 (2005) (Hybrid Assistive Limb) (https://www.cyberdyne.jp/english/products/HAL/index.ht $\mathrm{ml}$, 2018) estos robots que originalmente fueron desarrollos para mejorar la calidad de vida de personas con capacidad disminuidas fueron pensados como una entidad mecánica artificial (antiguamente llamados autómatas) y un sistema electromecánico conducido por programas de computadora. De la misma manera el desarrollo de la ortesis y las extremidades robóticas, etc.

Mientras que en los Moluscos (Hickman, 2006) existe un manto protector o palio, es un doble pliegue tegumentario que se extiende por toda la pared dorsal del cuerpo que segrega la concha, esta tiene tres capas y su capa exterior está formada por la conquiolina y su función es de protección. En algunos bivalvos la constitución de la concha en edad adulta puede ser de entre 450 a 5000 capas delgadas y paralelas. Dentro de esta especie los más estudiados por los arquitectos han sido los gasterópodos pulmonados por su forma y han sido fuente de inspiración para varios proyectos de arquitectura.

En el caso del filo Anélidos (Hickman, 2006), tienen una amplia distribución mundial, esto permite deducir que al realizar una analogía con este filo puede ser replicado en cualquier ubicación del planeta, analizamos específicamente los gusanos segmentados con el potencial que poseen, esta especie es capaz de realizar un movimiento independiente y separado en cada segmento (septos), la repetición de sus partes (metámero) con un sistema de seguridad que permite el funcionamiento y la supervivencia aún si alguno de sus segmentos sufre algún tipo de contratiempo. El movimiento peristáltico que caracteriza a los gusanos segmentados como los analizados y emulados en el proyecto CMMWorm, también existe en el tejido de los órganos internos del ser humano. El movimiento en el caso de los anélidos se logra por la contracción y dilatación de los fuertes músculos circulares y longitudinales, recubiertos por una epidermis y una cutícula externa delgada, mientras que, en el caso del hombre, las contracciones y relajaciones son radialmente simétricas y son las fibras musculares lisas la que permiten este movimiento.

En el caso del hombre, el movimiento peristáltico se divide en tres tipos de ondas y su función es la de movilizar los alimentos a través del aparato digestivo; el movimiento opuesto se denomina antiperistáltico que es el que produce el reflujo. El estímulo para el movimiento se genera a partir del músculo liso y está mediado por el sistema nervioso autónomo o visceral (SNA) cuya función principal es la homeostasia. Esta última es la capacidad de mantener una condición interna estable compensando los cambios que ocurren en su entorno mediante el intercambio regulado de materia y energía o no, en un equilibrio dinámico con procesos reversibles que al mismo tiempo producen reacciones opuestas al mismo paso. La homeostasia que depende del sistema autónomo, tiene como centro de control al hipotálamo (computadora central) un sistema sensitivo con termo receptores viscerales y externos al hipotálamo y el hipotálamo mismo (sensores) y un sistema efector que en caso del hombre que se desarrolla con un proceso de vasodilatación y vasoconstricción, actividad muscular de contracción y relajación, temblor (en ambos casos un intercambio de energía), aceleración o disminución del metabolismo (intercambio de materia o químico) y cosas voluntarias sugeridas por la corteza cerebral como abrigarse o desabrigarse, tomar agua, etc. En el presente trabajo hemos descartado la opción de intercambio de materia o químico.

Respecto a los reptiles lo que nos interesa es que poseen una piel dura como un exoesqueleto, pero es de escamas queratinizadas (endurecidas), los caimanes cuyas escamas permanecen a lo largo de toda la vida crecen gradualmente para suplir el desgaste. En otros casos como serpientes y lagartos crecen nuevas escamas debajo de las antiguas y así son mudadas a intervalos.

En función al análisis realizado, para nuestra propuesta que se dirige especialmente a la construcción de una envolvente arquitectónica como caso de estudio que involucra el desarrollo del pensamiento proyectual que posea la inclusión de sistemas descentralizados y auto organizados. En dicha propuesta se ha indagado la inclusión de segmentos con movimiento independiente en analogía a los Anélidos, tomando como referencia el trabajo previo de CMMWorm, un sistema compuesto por paneles que puedan ser sustituidos cuya función y comportamiento simula a la de un exoesqueleto en semejanza a los Artrópodo, pero con características similares a las escamas de los Reptiles, sobre una malla trenzada, contará con segmentos accionados y otros con movimiento involuntario que seguirán a los anteriores. El comportamiento puede ser ejecutado por un robot con características similares al industrial y produciendo las agitaciones de manera coordinada en semejanza con el meneo en los Anélidos. Imaginamos así la aplicación de estos brazos robóticos en cada articulación de la malla exterior trenzada del exoesqueleto del edificio, compuesto por una serie de segmentos rígidos unidos por articulaciones. Una superficie NURBS doble que genere el trenzado de la malla y donde se produce el imbricado de las placas a modo de escamas o exoesqueleto. Una computadora por segmentos que controle los motores de paso individuales conectados a cada junta (axón o neurita) como un sistema nervioso central pero parcial a la vez para que tenga lugar la actividad del sistema nerviosos autónomo (SNA) y la utilización de la hidráulica o la neumática para generar el movimiento, el SNA de la envolvente fue pensado con sensores fotosensibles, sensores de termo sensibles y sensores de movimiento para establecer la necesidad de movimiento y hacia donde, como parte del sistema nervioso ganglionar ventral que se da en el caso de los Artrópodos y en el hombre, con la función en este último de mantener la homeostasia. Todo el conjunto a manera de sistema descentralizado y auto organizado genera la transformación automática de la envolvente arquitectónica. La forma de la arquitectura se proyecta en reposo y como punto de arranque del sistema, pero una vez puesto en funcionamiento la forma la decide en total libertad el sistema (salvo los límites de anclaje del sistema mismo). Este sistema que es disidente en su forma, está pensado como replicable pero no en su forma misma que será exclusiva, sin posibilidad de producción masiva y a pesar de actuar como una envolvente autónoma, puede ser dirigido en algunos casos por el usuario con el uso de dispositivos, pudiendo generar 
formas de una arquitectura colectiva. Su apariencia de infinito, la ruptura de los límites a través de los posicionamientos más diversos dota al modelo propuesto de una integración homeostática con el entorno urbano, que predica un mensaje universal y que trasciende a culturas y países.

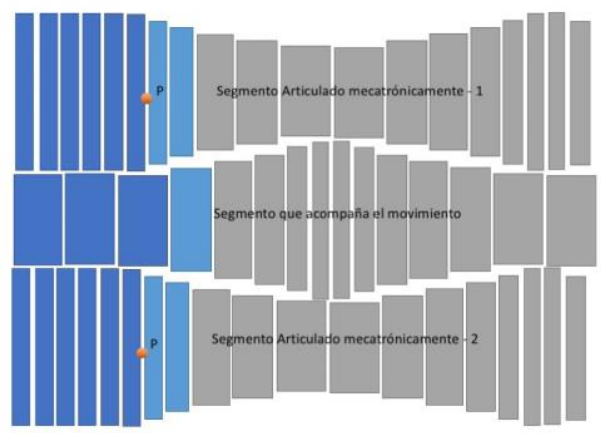

Figura 14: Propuesta vista de frente - Fuente: Elaboración propia

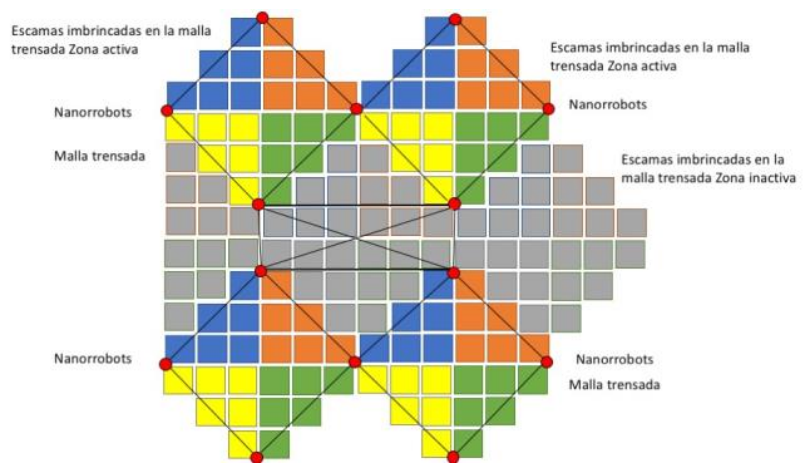

Figura 15: Propuesta vista de frente - Fuente: Elaboración propia

La segmentación propuesta permitirá además el recambio de partes de la envolvente. Ya que es de pensar que los materiales con los que se construya la envolvente arquitectónica como cutícula son de corta duración o bien la envolvente arquitectónica es de corta duración. Ya que el filo dentro de sus características posee la muda del exoesqueleto. $\mathrm{Y}$ las experiencias previas del proyecto CMMWorm con 3D-Printer que relacionan el modelo de neurociencia computacional de Wilso-Cowan para su desarrollo, dicho modelo considera una población homogénea de neuronas interconectadas del subtipo excitadoras e inhibidoras y que ha sido desarrollada como la hipersincronización de la actividad neurofisiológica a nivel global. Siguiendo el ejemplo del proyecto antes mencionado que toma como base el modelo WilsonCowan para generar el desplazamiento de puntos.

Otros cambios sugeridos son los referirlos a las circulaciones e instalaciones, a las que imaginamos como un sistema abierto y el sistema de ventilación puede ser mediante la superficie de la envolvente análoga al exoesqueleto autómata y utilizando técnicas de ventilación natural.

\section{RESULTADOS}

Como consecuencia de esta investigación podemos inferir que en la actualidad podemos construir un pensamiento proyectual de la envolvente arquitectónica con los elementos que describimos en secciones anteriores y con sus característica, pero sugerimos que con el avance del desarrollo de las tecnologías emergentes y con componentes cercanos a la escala nono métrica, las envolventes arquitectónicas que fueran diseñadas para emular los sistemas y el comportamientos de los ejemplares del reino animal y los artrópodos (Figura 16), podrán disminuir la escala de los segmentos que lo forman, aumentando la cantidad de articulaciones y sustituyendo los brazos robóticos con comportamiento de robots industriales con los nanorrobots 0 nanobots (Figura 17). Al ser de tamaño tan reducido se necesitaría gran cantidad, un enjambre de nanobots como una niebla útil (Fog, 2001) y los segmentos se reducirán en tamaño, pero aumentarán en cantidad, permitiendo que las formas arquitectónicas

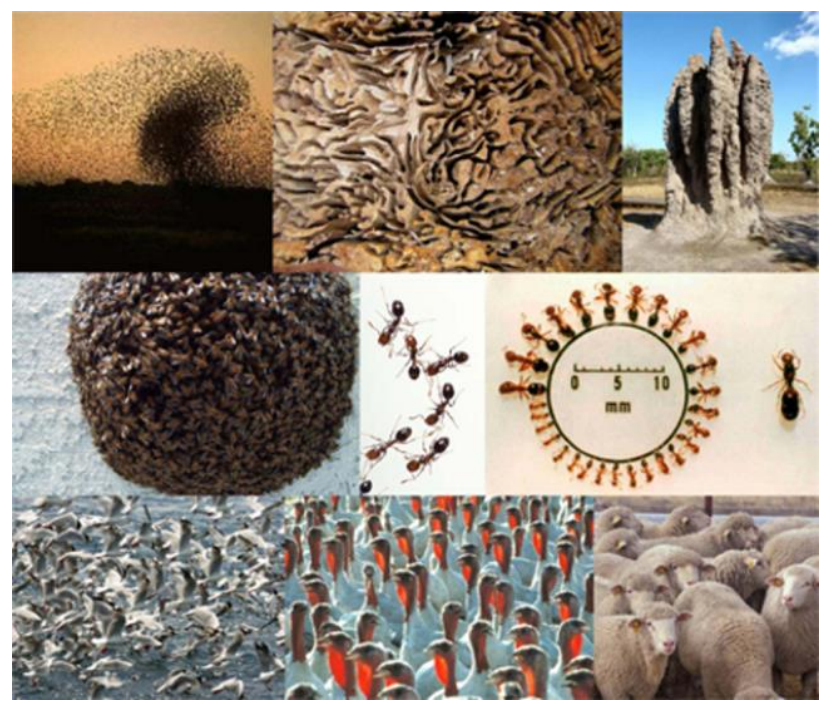

Figura 16: Enjambres - Fuente:

http://www.galacticsuitedesign.com/blogs/GSmoonrace/?p=102

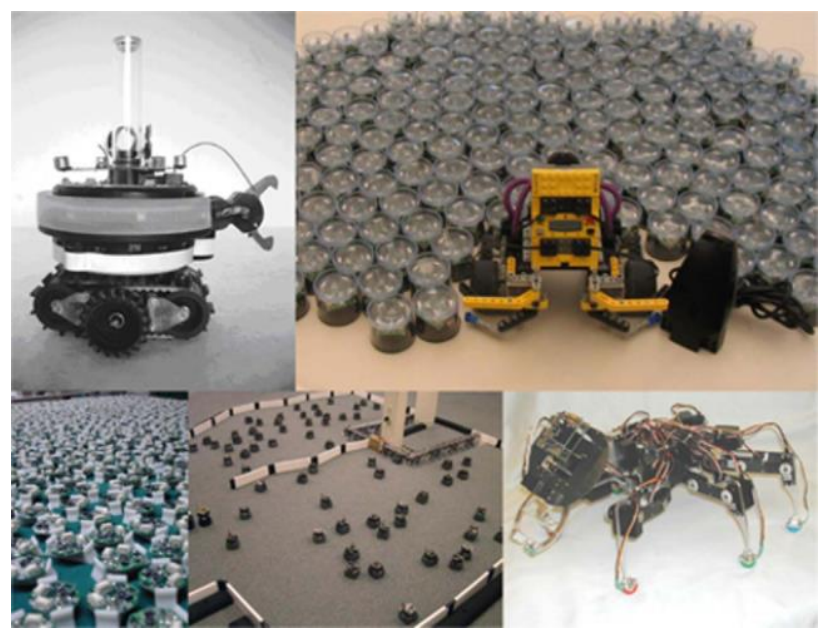

Figura 17: Robótica de enjambre - Fuente:

http://www.galacticsuitedesign.com/blogs/GSmoonrace/?p=102

puedan generar movimientos en varios ejes y la resultante puede permitir movimientos orgánicos y ondulantes (Boxerbaum, A., et al, 2012). Convirtiendo a la arquitectura en blanda y peluda (Estévez,1015). En esta investigación estamos trabajando en el tercer nivel de la biomimética (Benyus, 2009); estamos diseñando y proyectando por analogía a los sistemas y conductas naturales. 


\section{DISCUSIÓN}

En esta propuesta y en la actualidad existe un inminente límite físico entre el diseño como pre-figuración intelectual que se propone y la concreción física; también un límite de conocimiento de parte de los autores hacia otras áreas del conocimiento que hace necesaria la integración con especialistas de otras áreas disciplinares para continuar con la investigación y existe un límite económico para el desarrollo de las investigaciones, común a varios países latinoamericanos. Pero ninguno de los tres límites son determinantes para el abandono de las investigaciones que pasan a la etapa de prototipo.

\section{BIBLIOGRAFÍA}

https://www.cyberdyne.jp/english/products/HAL/index.html. (2018). What's HAL? Retrieved from Cyberdyne: https://www.cyberdyne.jp/english/products/HAL/index.html

University, W. R. (2011). Biorobots. Retrieved from Biologically Inspired Robotics: http://biorobots.case.edu/projects/softworm/

Boxerbaum, A., Horchler, A., Shaw, K., Chiell, H. \& Quinn, R. (2012) Continuous wave peristaltic motion in robot. The International Journal of Robotic Research, 31, 3, 302-318. doi:10.1177/0278364911432486
Estévez, A. (2015). Arquitectura Biodigital y Genética - Escritos. Barcelona: Bubok Publishing S.L.

Estévez, A. (1-5 de 12 de 2008). Arquitectura Biodigital. Recuperado el 22 de 06 de 2018, de Comunicades: http://cumincades.scix.net/cgi-

bin/works/Show?_id=sigradi2008_172\&sort=DEFAULT\&sear ch $=$ Estevez\&hits $=2$

Benyus, J. (2009). Biomimicry - Innovation Inspired by Nature. New York: HarperCollings e-books.

Granero, A. (2016). El espacio arquitectónico topológico digital. SIGraDi 2016, XX Congreso de la Sociedad Ibero-americana de Gráfica Digital (págs. 693-699). Buenos Aires: UBA, FADU - Blucher.

Hickman, e. a. (2006). Principios integrales de zoología. Madrid, España: McGraw-Hill/Interamericana de España.

Schumacher, P. (1 de 6 de 2011). La Autopoesis de la Arquitectura. Recuperado el 22 de 06 de 2018, de Universidad de Chile: http://www.revistas.uchile.cl/index.php/RA/issue/view/2430

Utility Fog: The Stuff that Dreams Are Made Of by J. Storrs Hall Originally published 1993 Published on KurzweilAI.net July 5, 2001.

Wilson, H., Cowan, J. (1972) Excitatory and Inhibitory Interactions in Localized Populations of Model Neurons. Recuperado 22 de 06 de 2018, from Biology Journal 